\title{
L'ÉRISMATURE ROUSSE (OXYURA JAMAICENSIS) : UNE INTRODUCTION PROBLEMMATIQUE D'OISEAU DANS LES MILIEUX AQUATIQUES.
}

\author{
C. PERENNOU, \\ avec la collaboration technique de P.J. DUBOIS et B. HUGHES.
}

Station Biologique de la Tour du Valat, Le Sambuc, 13200 Arles, France.

\section{RÉSUMÉ}

Introduit accidentellement en Grande-Bretagne dans les années 1950, ce canard d'origine américaine y est aujourd'hui en expansion constante. II a, dans les années 1970, commencé à peupler le continent européen, en particulier la France et l'Espagne où il se reproduit désormais. Cette expansion d'une espèce d'oiseau aquatique introduite, en France et dans le reste de l'Europe continentale, pose de graves problèmes pour la conservation à long terme d'une espèce proche, considérée comme menacée à l'échelle mondiale, l'Érismature à tête blanche (Oxyura leucocephala), en raison de :

- l'hybridation avec l'espèce introduite ;

- la compétition sur les terrains d'alimentation ;

- la remise en cause des programmes de conservation (Espagne) et de réintroduction (France, Italie) de l'espèce européenne menacée.

L'historique de cette introduction, de son impact, de sa perception par les autorités et les associations, puis des mesures prises pour lutter contre celle-ci, est analysé, et des enseignements sont tirés pour d'autres espèces d'oiseaux aquatiques introduits en France et en Europe.

\section{THE PROBLEM OF THE INTRODUCTION OF AN AQUATIC BIRD, THE RUDDY DUCK (OXYURA JAMAICENSIS).}

\section{SUMMARY}

This North American duck was accidentally introduced into Britain in the 1950's and is presently expanding rapidly in this country. In the 1970's, it started colonizing continental Europe, especially France and Spain where it now breeds.

This expansion in France and the rest of Europe causes serious conservation concern for a related species, the White-headed Duck (Oxyura leucocephala), a globally threatened species :

- hybridization with the introduced species ;

- competition on feeding grounds ;

- threats to existing conservation (Spain) or reintroduction (France, Italy) projects. 
The history of this introduction, of its impact, of its perception by the authorities-incharge and NGOs, and finally of action taken, is analysed first. Then, lessons are drawn for other bird species introduced into France and Europe.

\section{INTRODUCTION : LE CONTEXTE}

\section{Les deux espèces}

L'Érismature rousse Oxyura jamaicensis est un canard nord-américain introduit accidentellement en Grande-Bretagne en 1953, à partir d'individus échappés de parcs zoologiques, notamment du Wildfowl Trust à Slimbridge (Gloucestershire). Son expansion fut rapide : il y nicha dès 1960 . Sa population actuelle compte environ 3500 oiseaux et 600 couples nicheurs, et elle croît au rythme annuel de $8 \%$ (HUGHES, sous presse). Très vite, l'espèce tente de s'installer en Europe continentale : elle a été observée en France dès 1974, en Espagne dès 1983. En France, 9 individus en moyenne sont observés chaque année, avec des groupes records de 16 oiseaux ensemble en 1984, 13 en 1983, et 9 en 1992 et 1994 (selon le Comité d'Homologation National). Plus de 600 observations dans 20 pays du Paléarctique Occidental ont déjà été effectuées hors d'Angleterre (HUGHES, sous presse).

En Espagne, existe par ailleurs la dernière population européenne d'Érismature à tête blanche Oxyura leucocephala, espèce méditerranéenne et asiatique. Cette espèce est menacée à l'échelle mondiale (COLLAR et al., 1994) : moins de 19000 oiseaux survivent, dispersés de l'Espagne et de l'Afrique du Nord jusqu'en Mongolie. En Espagne, d'importants efforts entrepris par les autorités officielles et les associations ornithologiques ont permis le redressement des effectifs à près de 800 oiseaux en 1992, à partir d'une population tombée à 22 individus en 1977 (GREEN et ANSTEY, 1992 ; HUGHES, sous presse). Dans le sud de l'Espagne, l'espèce est devenue un symbole de succès en matière de protection des oiseaux.

Cette espèce nichait aussi en France jusqu'en 1966, en Corse (THIOLLAY, 1967). Elle a disparu depuis, vraisemblablement victime d'un braconnage intensif. Une réflexion préalable à une réintroduction dans l'île est en cours (PERENNOU et CANTERA, 1993), et d'ici quelques années les conditions pourraient redevenir favorables à son retour dans notre pays.

\section{Le problème}

L'Érismature rousse a commencé à se reproduire en Espagne à la fin des années 1980 . Les observations des scientifiques espagnols et anglais sur les oiseaux en liberté et en captivité ont montré que l'espèce américaine est extrêmement agressive envers tous les oiseaux d'eau (ARENAS et TORRES, 1992 ; HUGHES, 1992), en particulier envers l'Érismature à tête blanche. Elle la chasse de ses territoires d'alimentation, s'avère plus compétitive lors de la reproduction en s'accaparant les femelles de l'espèce autochtone, et produit ainsi des hybrides féconds.

Cette hybridation et cette compétition sont susceptibles de conduire à terme l'espèce méditerranéenne à la disparition par " dilution génétique ". Plusieurs espèces ou races d'oiseaux se sont déjà éteintes dans le monde de cette façon, ou sont sur le point de l'être : le grèbe Tachybaptus rufolavatus à Madagascar, une tourterelle dans l'Océan Indien, plusieurs races insulaires de canards... (COLLAR et al., 1994 ; HUGHES, sous presse). Le risque est donc bien réel de voir une espèce abondante en Amérique (au moins 660000 individus, trente fois plus que l'espèce eurasiatique ; ROSE et SCOTT, 1994) éliminer une espèce menacée d'Espagne, puis d'Afrique du Nord et du Moyen-Orient. Des Érismatures rousses y ont déjà été vues, notamment au Maroc.

\section{PRISE DE CONSCIENCE DU RISQUE ET ATTITUDES FACE À CELUI-CI : HISTORIQUE}

\section{2-1990 : un danger perçu comme " à long terme "}

La première mention apparente du risque que l'espèce exogène faisait courir à l'Érismature européenne apparaît dans une publication du Bureau International de 
Recherches sur les Oiseaux d'Eau (BIROE, devenu depuis Wetlands International) : dès 1982, ROOTH et SCOTT mentionnent explicitement le double danger (compétition et hybridation), en précisant qu'il préoccupe déjà sérieusement le monde de la conservation. Le problème semble ensuite oublié à l'échelle internationale. ANSTEY (1989) le mentionne comme d'importance limitée en Espagne, quoique nécessitant à long terme une attention spéciale. Pourtant, l'espèce est observée quasi-annuellement dans le pays, en nombres croissants (TORRES et al., 1994).

\section{1-1993 : le problème au premier plan}

Le problème réapparaît de façon aiguë en Espagne en 1991, lorsque la prédiction de ROOTH et SCOTT (1982) se vérifie : I'hybridation in natura a commencé. Des réunions techniques conduisent au démarrage immédiat d'un programme d'élimination des hybrides ainsi que des Érismatures rousses.

Parallèlement, une campagne espagnole de lobbying international aboutit à l'organisation par le BIROE, en mars 1993, d'une première réunion internationale consacrée au problème, 11 ans après sa première mention. Cinquante-quatre experts représentant 10 pays y recommandent un plan global de 38 mesures, destiné à " arrêter et renverser la tendance à l'expansion de l'Érismature rousse, de manière à garantir la survie de l'Érismature à tête blanche, espèce mondialement menacée " (IWRB, 1993). Elles concernent suivi, recherche sur les moyens de contrôle, contrôle, action en direction des éleveurs...

\section{3-1996 : des actions très inégales}

Les différents pays concernés en tant que lieu d'origine (Grande-Bretagne), de transit (Europe continentale) ou de risque concret (Espagne) prennent des attitudes et des mesures très différentes.

Le principal pays concerné, l'Espagne, poursuit le tir des Érismatures rousses et des individus hybrides afin de garantir la conservation d'une espèce très menacée, l'Érismature à tête blanche. Au total, 48 individus sont éliminés de 1991 à juin 1994, soit la quasi-totalité des oiseaux estimés " dangereux " (TORRES et al., 1994). Un guide d'identification des deux espèces d'Érismatures et de leurs hybrides est publié en Espagne (URDIALES et PEREIRA, 1993) ; il est bilingue (espagnol - français), afin de faciliter l'extension technique du contrôle à la France.

En Grande-Bretagne, se pose un problème de sensibilisation du public, désormais habitué à l'espèce introduite et, par tradition insulaire, peu sensible aux problèmes qu'elle peut causer ailleurs. Les autorités gouvernementales concernées et les ONG, convaincues rapidement du problème, forment dès 1992 un groupe de travail sur le problème et entreprennent une campagne de sensibilisation du grand public soigneusement pensée. Parallèlement, elles mettent en place des recherches de terrain visant à étudier l'efficacité des mesures de contrôle (HUGHES, sous presse). Après 3 années de recherche, un programme de contrôle à l'échelle régionale (sur 2 provinces) est lancé en 1995 (HUGHES, comm. pers.), avec pour objectif d'éliminer le maximum d'oiseaux et de maintenir ainsi l'émigration vers le continent à un niveau minimal. Par ailleurs, un système visant à dissuader les éleveurs de détenir en captivité des Érismatures rousses y est en cours d'élaboration. La commercialisation de l'espèce y nécessite déjà une autorisation spéciale.

Lors d'un second atelier international en septembre 1994 à Cordoue (Espagne), les actions entreprises en Espagne et en Grande-Bretagne sont louées, mais les participants jugent "particulièrement inquiétante l'absence de décision en Hollande, Belgique, Allemagne, Islande, France, Irlande et Maroc " (ANONYME, 1994a). Pourtant, la Communauté Européenne a recommandé à tous ses états membres d'encourager les mesures de contrôle de l'Érismature rousse (ANONYME, 1994a). 


\section{CAS PARTICULIER DE LA FRANCE}

\section{Position géographique particulière}

La France se situe entre l'importante population britannique d'Érismatures rousses et l'Espagne, dernier refuge européen de l'Érismature à tête blanche. Cette position charnière lui donne une grande importance quant aux mesures de contrôle à y prendre. Bien que certains individus observés en France aient pu s'échapper de captivité, l'origine anglo-saxonne de la grande majorité des oiseaux est fortement suggérée par leur concentration dans le nord-ouest de la France (Figure 1 ; d'après les données du Comité d'Homologation National (C.H.N.)).

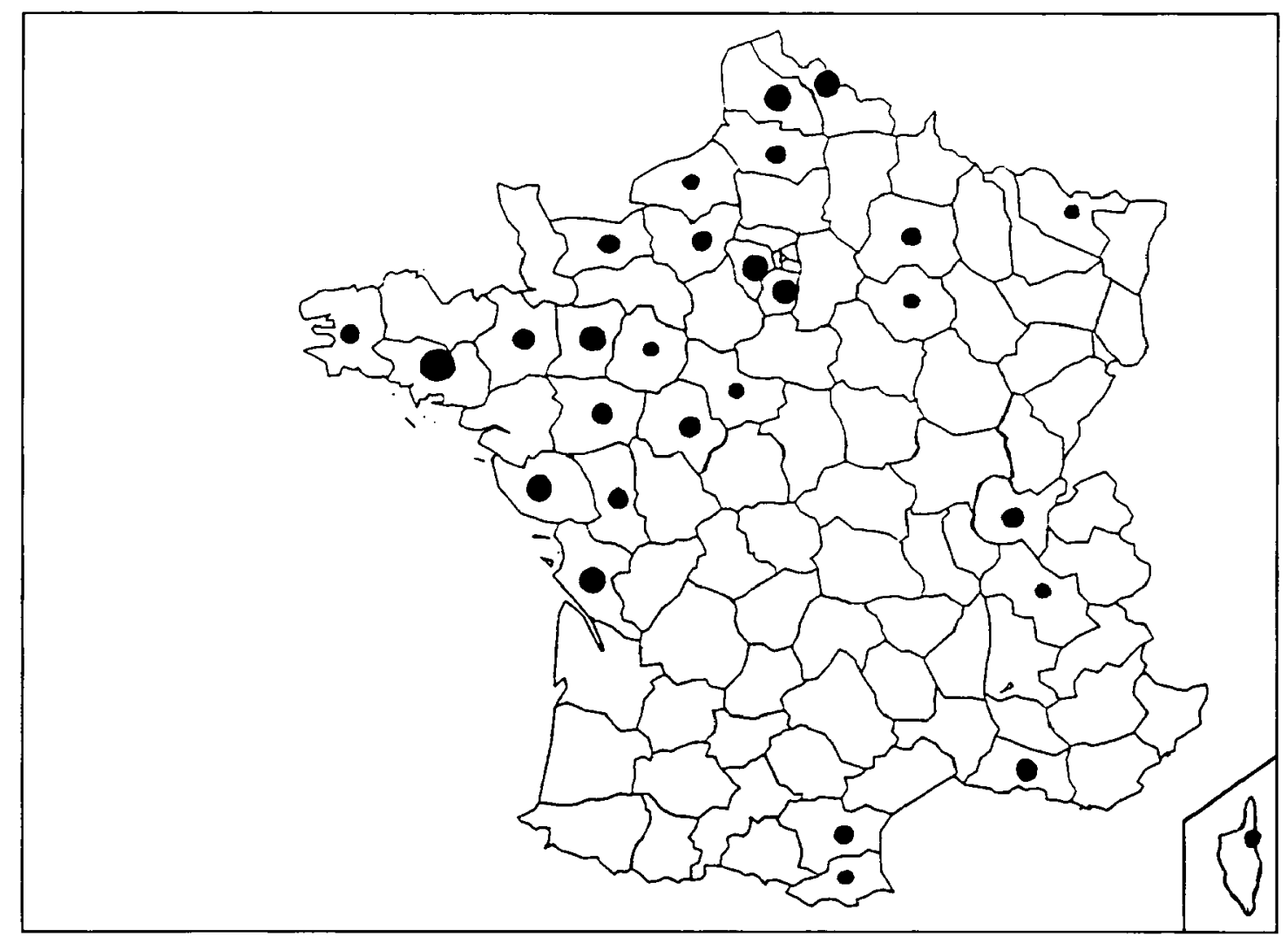

Figure 1

Distribution géographique des observations d'Érismature rousse en France (1974-1994). Données du Comité d'Homologation National (une observation = 1, quel que soit le nombre d'individus vus).

Figure 1

Geographical distribution of ruddy duck observations in France between 1974-1994. Data from the French Rarities Committee (one observation $=1$, whatever the number of birds seen).

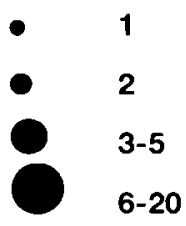

observation

observations

observations

observations

Un second indice est leur concentration dans le temps, principalement en hiver dès novembre (Figure 2), période où les oiseaux anglais se regroupent pour hiverner sur quelques réservoirs. Enfin, le nombre d'observations en Europe en général est fortement corrélé à l'augmentation des effectifs britanniques (Figure 3 ; d'après HUGHES, sous presse). Sans le démontrer strictement, ces trois éléments convergents indiquent que l'émigration britannique est vraisemblablement la source principale des observations continentales. 


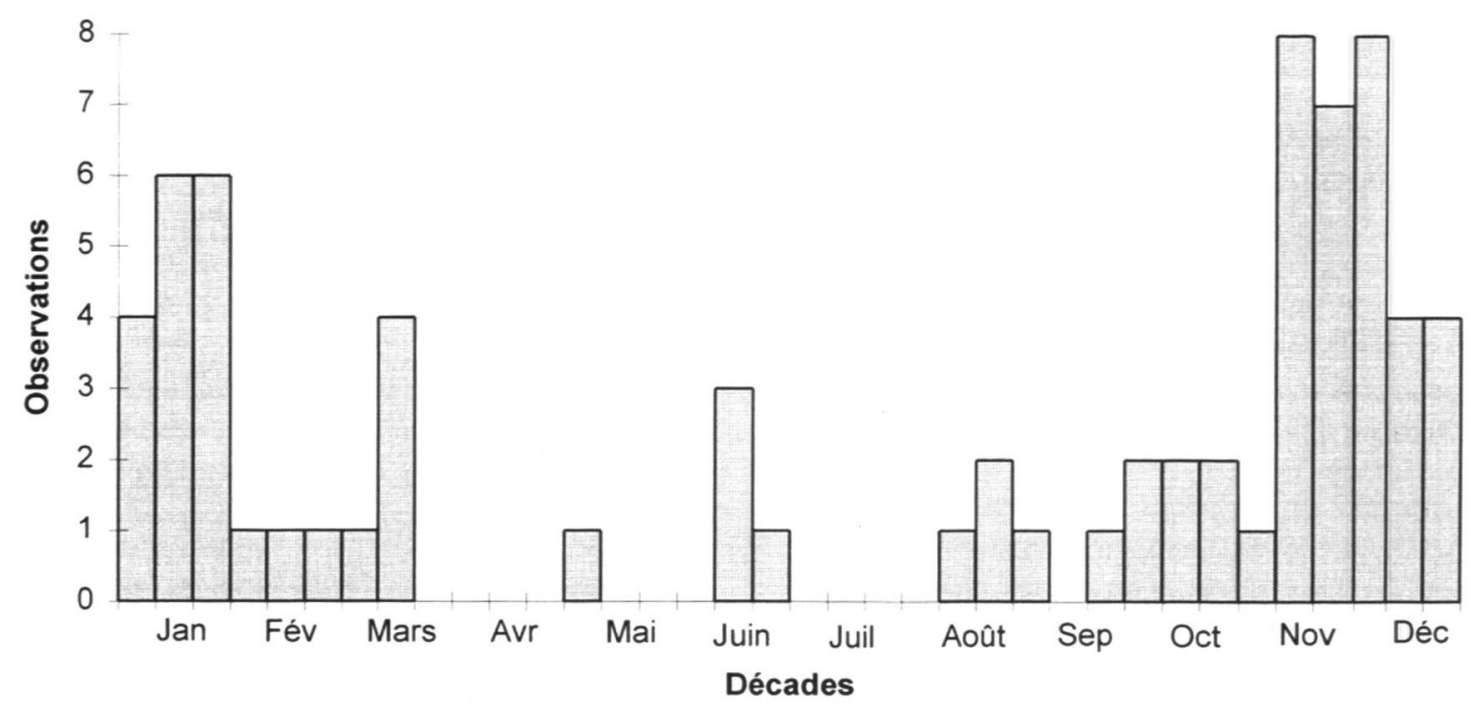

Figure 2

Distribution par décades des observations d'Érismature rousse en France (1974-1994). Données du Comité d'Homologation National.

\section{Figure 2}

Distribution in time (10-days periods) of ruddy duck observations in France between 1974-1994.

Data from the French Rarities Committee.

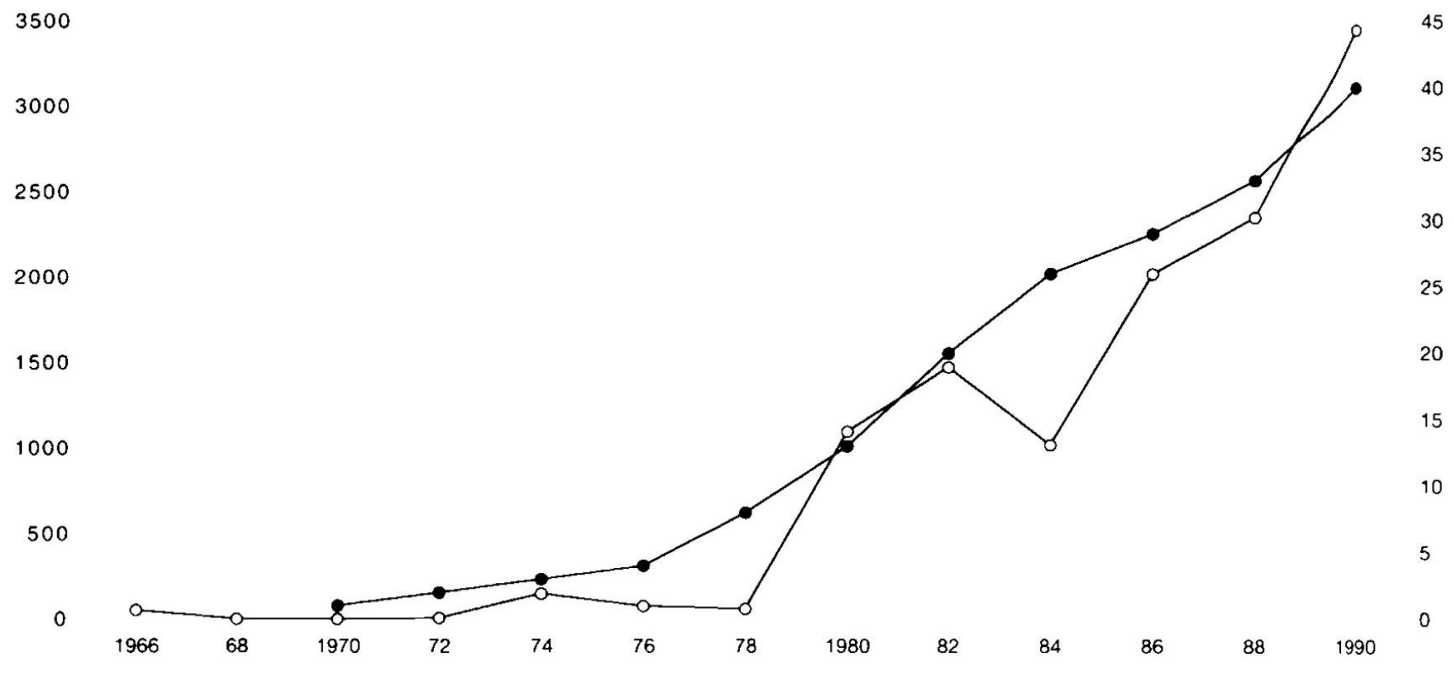

Figure 3

Augmentation parallèle des observations d'Érismature rousse sur le continent européen et de sa population britannique (d'après HUGHES, sous presse).

Figure 3

Parallel increase of ruddy duck observations in continental Europe and of its British population (after HUGHES, in press).

- Estimation de la population britannique (échelle de gauche). British population estimate (left scale).

Nombre d'observations sur le continent (échelle de droite). Number of continental records in Europe (right scale). 
L'Érismature rousse a niché en France dès 1988. La discrétion de l'espèce a empêché de prouver la reproduction par la suite (C.H.N., 1981-1994). Cependant, des stationnements printaniers (couples, groupes) sont régulièrement observés dans quelques régions d'étangs (Dombes, Loire-Atlantique, Vendée, Mayenne, Pas-de-Calais ; C.H.N., 1981-1994), et la reproduction, au moins occasionnelle, y est donc possible.

\section{3-1994 : renversement de la perception du problème}

L'attention du public naturaliste semble attirée pour la première fois sur ce problème en 1993 (PERENNOU et GREEN, 1993), dans la revue l'Oiseau Magazine. Cependant, les associations restent opposées, par principe, à tout contrôle ; la Ligue pour la Protection des Oiseaux (LPO) rappelle, dans le même numéro de ce magazine, son opposition totale. II est alors considéré que "l'action à entreprendre se [situe] principalement en Grande-Bretagne " (ROCAMORA, 1993). En 1994, un premier article paraît dans la revue avicole Aviornis International (ANONYME, 1994b). II vise à sensibiliser les éleveurs francophones de l'espèce, afin d'éviter de nouvelles évasions.

Fin 1993, une ètude de faisabilité pour la réintroduction de l'Érismature à tête blanche en Corse (PERENNOU et CANTERA, 1993) liste les conditions préalables à remplir avant toute réintroduction, notamment le contrôle de l'Érismature rousse. En effet, sans ce contrôle, une réintroduction risquerait à terme de donner naissance à une population hybride, l'espèce américaine ayant déjà été observée sur le site prévu de réintroduction.

Cette étude est diffusée en 1994 à toutes les institutions concernées. Elle semble avoir forcé à la réflexion. En septembre 1994, l'Office National de la Chasse confirme la nécessité de ce contrôle ; le même mois, la LPO adopte une nouvelle position, favorable au contrôle sous des conditions bien définies. En décembre 1994, un petit groupe de travail national est constitué, sous l'égide du Ministère de l'Environnement. II comprend associations, scientifiques, instances administratives: Ministère, CNPN, LPO, Muséum, Tour du Valat, ONC. C'est donc à la fin 1994 qu'un consensus se dégage sur le caractère dangereux de cette introduction involontaire, et sur la nécessité d'un contrôle.
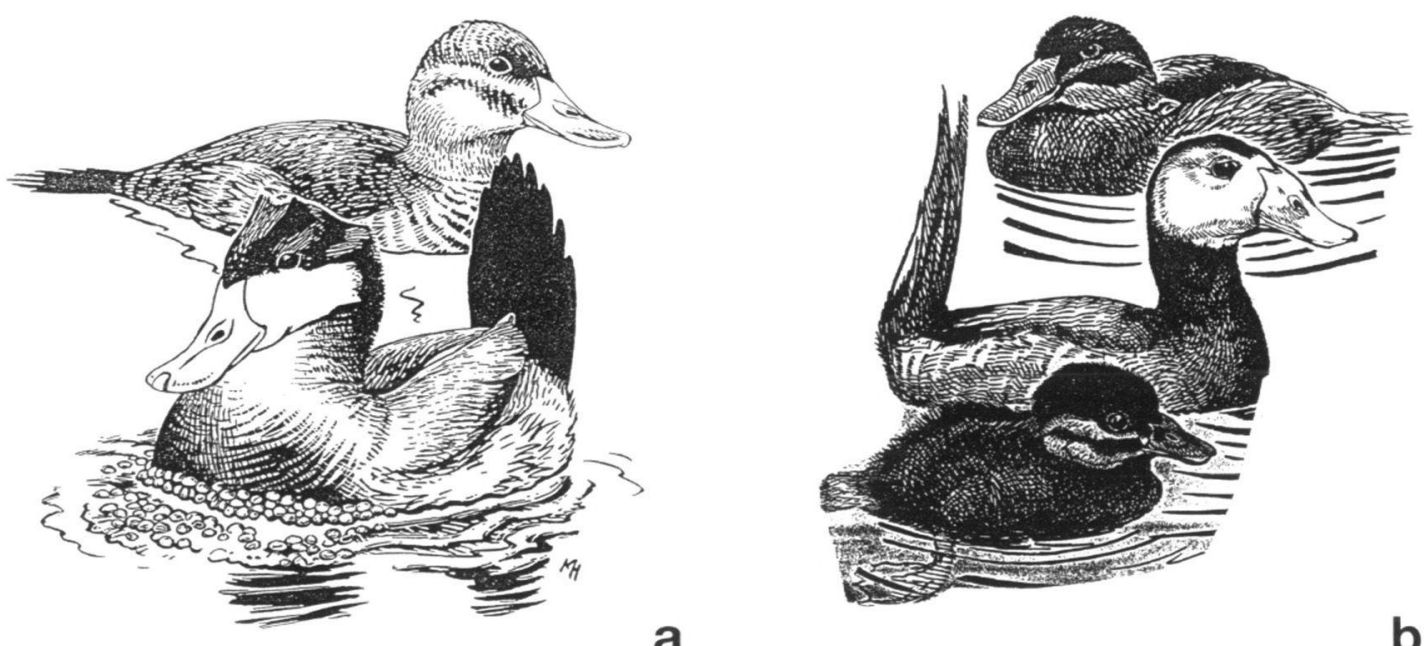

a

b

\section{Figure 4}

Érismature rousse (4a) et Érismature à tête blanche (4b). Dessins de Mark HULME et Joe BLOSSOM (Wildfowl and Wetlands Trust).

\section{Figure 4}

Ruddy Duck (4a) and White-headed Duck (4b). Drawings by Mark HULME and Joe BLOSSOM (Wildfowl and Wetlands Trust). 


\section{Mesures en cours}

Ce groupe définit, début 1995, quatre directions majeures d'actions :

- sensibilisation du public, notamment associatif : un texte agréé par tous fut rapidement rédigé à cet effet ;

- examen du statut juridique de l'Érismature rousse, et éventuellement modification pour l'adapter aux besoins du contrôle ;

- mise en place d'un réseau de contrôle agréé par le milieu associatif et par l'ONC;

- sensibilisation des éleveurs d'oiseaux captifs, et dissuasion de la détention de l'espèce, notamment par une interdiction de son commerce.

Cependant, de février 1995 à février 1996, aucun progrès n'eut lieu. Selon le Ministère, le climat très tendu entre monde de la chasse et associations de protection à propos d'autres questions (chasse au bruant ortolan Emberiza hortulana; modification de la Directive Européenne "Oiseaux "...) empêchait toute application d'un accord pourtant unanime sur l'Érismature rousse : recommander l'éradication par le tir d'une espèce d'oiseau aurait été perçu comme une provocation. En février 1996, néanmoins, le Ministère était confiant que la sensibilisation du monde associatif, ainsi que les décisions ministérielles nécessaires, allaient pouvoir se mettre en place au cours du premier semestre 1996.

\section{CONCLUSIONS}

Les quatorze années écoulées depuis la première mention du problème potentiel permettent de tirer les conclusions suivantes:

1) Même lorsque le problème est clairement pressenti, mais ne se pose pas encore en termes réels (1982-1990, dans notre cas), rien n'est tenté pour l'éviter. Or, à ce stade, une action serait théoriquement plus facile, car les effectifs introduits sont alors plus faibles. On pourrait accuser de manque de clairvoyance les organisations, gouvernementales ou non, chargées de ces questions. Ce serait cependant oublier que, par manque de moyens dans le domaine de la protection de la nature, seules les questions urgentes sont habituellement traitées, et des problèmes futurs importants, même entrevus, ne peuvent qu'être négligés.

2) Face à des oiseaux capables de grands déplacements rapides, seule une action concertée internationale peut contrecarrer une introduction.

Dans notre cas, elle se met difficilement en place. La réaction la plus rapide fut celle du pays touché directement par les effets de l'introduction (Espagne), suivi du pays responsable (Grande-Bretagne). Les pays tiers (dont la France) tendent à réagir beaucoup plus lentement, n'étant pas touchés directement et ne s'estimant pas responsables. En ce sens, le projet de réintroduction de l'Érismature à tête blanche a pu faire effet de catalyseur en France, en plaçant le pays dans une situation similaire à l'Espagne : une population (encore potentielle) d'Érismatures à tête blanche, menacée d'hybridation.

II faut pourtant noter qu'à défaut d'être responsable de l'introduction initiale, la France. de par sa position géographique, pourrait être responsable de son éventuelle expansion géographique, si s'installait une population nicheuse de l'espèce américaine.

3) Les cas d'oiseaux aquatiques introduits se reproduisant en France sont encore rares, par rapport à d'autres pays comme la Grande-Bretagne : en dehors de l'Érismature rousse, ils concernent surtout la Bernache du Canada Branta canadensis, l'lbis sacré Threskiornis aethiopicus en sud-Bretagne, et le Flamant du Chili Phoenicopterus chilensis occasionnellement en Camargue (YEATMAN-BERTHELOT et JARRY, 1994).

Au contraire de nombreuses autres espèces (plantes, poissons, invertébrés...), les oiseaux aquatiques semblent surtout susceptibles d'impacts sur des espèces proches 
(hybridation ou compétition), plutôt que d'impacts plus larges sur les écosystèmes aquatiques.

4) Prévoir l'impact potentiel de telles introductions sur les espèces autochtones est très difficile. Une première approche du problème pourrait être de sérier les probabilités d'impact d'une espèce introduite sur les populations autochtones, selon les critères suivants:

- il existe une espèce congénérique se reproduisant régulièrement dans le même pays - risque élevé d'hybridation, de compétition dans le pays ;

- il existe une espèce congénérique se reproduisant régulièrement dans la même région biogéographique - risque élevé d'hybridation, de compétition dans les pays proches ;

- il existe une espèce proche, mais non congénérique, se reproduisant régulièrement dans la même région biogéographique , risque modéré d'hybridation, mais compétition potentielle.

Le caractère migrateur (ou nomade) de l'espèce introduite, ainsi que son grégarisme, sont d'autres facteurs susceptibles d'accélérer l'accès d'individus exogènes aux zones de reproduction des espèces autochtones, et donc d'augmenter le risque d'hybridation.

\section{POST SCRIPTUM}

Le Ministère de l'Environnement Français a pris le 12 novembre 1996 un arrêté autorisant la destruction par tir de l'Érismature rousse (J.O. du 28 novembre 1996), et a approuvé le 9 octobre 1996 un plan d'action contre l'Érismature rousse, sur la base des propositions du Groupe de Travail constitué en 1994. La première réunion du comité chargé d'appliquer ce plan a eu lieu à Paris le 15 janvier 1997.

\section{REMERCIEMENTS}

Je souhaite ici remercier particulièrement A. GREEN (Estacion Biologica de Doñana), G. ROCAMORA (LPO), P.J. DUBOIS (Comité d'Homologation National (CHN)), J.P. CANTERA (AGENC), B. HUGHES (Wildfowl and Wetlands Trust), S. PAUL (Ministère de l'Environnement), E. ROSECCHI, J. SKINNER et J. JALBERT (Station Biologique de la Tour du Valat), et deux lecteurs anonymes pour leurs commentaires pertinents et / ou leur aide dans la recherche d'informations.

\section{BIBLIOGRAPHIE}

ANONYME, 1994a. International Technical Meeting on Oxyura leucocephala and Oxyura jamaicensis in the Palearctic Region. Córdoba (Spain), 29-30 sept. 1994, Conclusions and recommendations, $8 \mathrm{p}$.

ANONYME, 1994b. Nouvelles de Slimbridge. Aviornis International, 116, 14-19.

ANSTEY S., 1989. The Status and Conservation of the White-headed Duck Oxyura leucocephala. IWRB Special Publication $n^{\circ}$ 10, Slimbridge (Great Britain), $127 \mathrm{p}$.

ARENAS R., TORRES J.A., 1992. Biologia y situacion de la Malvasia en España. Quercus, 73, 14-21.

C.H.N. (Comité d'Homologation National), 1981-1994. Observations des espèces soumises à homologation en France. Alauda, divers volumes.

COLLAR N.J., CROSBY M.J., STATTERSFIELD A.J., 1994. Birds to Watch. 2 - The World list of Threatened Birds. BirdLife Conservation Series $n^{\circ} 4$, Cambridge, $407 \mathrm{p}$.

GREEN A.J., ANSTEY S., 1992. The status of the White-headed Duck Oxyura leucocephala. Bird Conservation International, 2, 185-200. 
HUGHES B., 1992. The ecology and behaviour of the Ruddy Duck Oxyura jamaicensis in Great Britain. Ph. D. Thesis, University of Bristol (Great Britain), $233 \mathrm{p}$.

HUGHES B., sous presse. The Ruddy Duck Oxyura jamaicensis in Europe and the threat to the White-headed Duck Oxyura leucocephala. Proc. IWRB Symposium "Anatidae 2000", Strasbourg, France, 5-9 dec. 1994.

IWRB, 1993. Summary and Recommendations of the International Oxyura jamaicensis workshop, Arundel (Great Britain), 1-2 March 1993. IWRB, Slimbridge (Great Britain), 6 p.

PERENNOU C., CANTERA J.P., 1993. Etude de faisabilité pour la réintroduction de l'Érismature à tête blanche Oxyura leucocephala sur l'étang de Biguglia, Haute-Corse. Rapport Tour du Valat, AGENC, 63 p.

PERENNOU C., GREEN A.J., 1993. Érismature contre Érismature. L'Oiseau Magazine, 31, 28-29.

ROCAMORA G., 1993. Compte rendu de l'atelier de travail international sur l'Érismature rousse, 1-2 mars 1993, Arundel (Great Britain). Rapport interne LPO.

ROOTH J., SCOTT D.A., 1982. The introduction of exotic species in SCOTT D.A., Managing Wetlands and their Birds, 279-283, IWRB, Slimbridge (Great Britain).

ROSE P.M., SCOTT D.A., 1994. Waterfowl population estimates. IWRB Publication $n^{\circ} 29$, Slimbridge (Great Britain), $102 \mathrm{p}$.

THIOLLAY J.M., 1967. Notes sur l'avifaune corse. Ois. Rev. fr. Orn., 37, 104-113.

TORRES J.A., MORENO B., ALCALA-ZAMORA A., 1994. Resultados del programa de control de la malvasia canela. Quercus, 104, 14-18.

URDIALES C., PEREIRA P., 1993. Clé d'identification de O. jamaicensis, O. leucocephala et ses hybrides. ICONA Publ., Madrid, $31 \mathrm{p}$.

YEATMAN-BERTHELOT D., JARRY G., 1994. Nouvel atlas des oiseaux nicheurs de France. Société Ornithologique de France, Paris, $776 \mathrm{p}$. 